\title{
Impacto del programa público en el control de diabetes
}

\author{
mellitus 2 en beneficiarios
}

Impact of the public program on the control of diabetes mellitus 2 in

beneficiaries

\section{Impacto del programa público}

\author{
Fabiola Katherine Bacusoy Palma, Lic. ${ }^{1}$ \\ Mabel Sánchez Rodríguez, Dra. ${ }^{2}$ \\ Estrella Mero Quijije, Lic. ${ }^{3}$ \\ Viviana Quiroz Villacreses, Lic. $^{4}$
}

${ }^{1}$ Ministerio de Salud Pública, Universidad Estatal del Sur de Manabí, Ecuador. Email: fabiola_bacusoy@hotmail.com

${ }^{2}$ Universidad Estatal del Sur de Manabí, Ecuador. Email; dramabelsan24@ hotmail.com

${ }^{3}$ Universidad Estatal del Sur de Manabí, Ecuador. Email; estre_25mar@ hotmail.com

${ }^{4}$ Universidad Estatal del Sur de Manabí, Ecuador. Email; vivi10_1989@ hotmail.com

Contacto: fabiola_bacusoy@hotmail.com

\section{Resumen}

La diabetes una enfermedad crónica considerada un problema de salud mundial, factor principal de enfermedades cardiovasculares con complicaciones muy temidas si no se maneja de forma adecuada, el autocuidado es esencial. En la actualidad el ministerio de salud pública maneja consigo un programa para diabéticos en los centros de atención primaria en salud. La presente investigación tiene como objetivo analizar el impacto de programa público en el control de diabetes mellitus 2 en los beneficiarios. Es un estudio de tipo descriptivo, analítico, retrospectivo que consiste en recopilar una gran cantidad de datos de acción rápida mediante la historia clínica de los pacientes, se estudió el impacto con una muestra de 40 usuarios para la investigación a quienes se les aplico la encuesta, test de findrisk y entrevista al personal. El $78 \%$ de ellos comprende pacientes de 60 años. Los resultados obtenidos demuestran que el control de la patología es inadecuado y el número de complicaciones alto relacionado con su insuficiente autocuidado pese a la asistencia al centro de salud de manera regular. Dentro de la muestra se aprecia que existe mayor riesgo en el género femenino que en el masculino y es por ello que se recomienda nuevas adaptaciones en la estructura del programa porque no se está cumpliendo los objetivos para el cual fue creado, todo en función de mejorar la calidad de vida de los diabéticos.

Palabras clave: problema de salud, sexo femenino, factores de riesgo, complicaciones

\section{Abstract}

Diabetes is a chronic disease considered a global health problem, a major factor in cardiovascular diseases with very feared complications if not managed properly, selfcare is essential. Currently, the public health ministry manages a program for diabetics in primary health care centers. The objective of this research is to analyze the impact of the public program on the control of diabetes mellitus 2 in the beneficiaries. It is a descriptive, analytical, retrospective study that consists of collecting a large amount of fast-acting data through the patients' clinical history. The impact was studied with a sample of 40 users for the research to whom the survey was applied, findrisk test and interview the staff. $78 \%$ of them comprise patients of 60 years. The results obtained show that the control of the pathology is inadequate and the number of complications is high related to its insufficient self-care despite the regular attendance at the health center. The sample shows that there is greater risk in the female than in the male gender and that is why new adaptations are recommended in the structure of the program because the objectives for which it was created 


\section{HIGIA DE LA SALUD}

are not being met, all in order to improve the quality of life of diabetics.

Keywords: health problem, female sex, risk factors, complications

\section{Introducción}

Según las estimaciones, de la Organización Mundial de la Salud (OMS) 422 millones de adultos en todo el mundo tenían diabetes en 2014, frente a los 108 millones de 1980. La prevalencia mundial (normalizada por edades) de la diabetes casi se ha duplicado desde ese año, pues ha pasado del $4,7 \%$ al $8,5 \%$ en la población adulta. Ello supone también un incremento en los factores de riesgo conexos, como el sobrepeso o la obesidad. En la última década, la prevalencia de la diabetes ha aumentado más deprisa en los países de ingresos bajos y medianos que en los de ingresos altos. (1)

Una de cada 11 personas en el mundo padece diabetes. Si los enfermos de diabetes fueran los habitantes de un solo país, sería el tercer país más grande del mundo, tan solo por detrás de China y la India y por delante de los Estados Unidos. (2)Se estima que se producen 3,7 millones de muertes que se provocan por la diabetes y una glucemia elevada, y se producen además 1,5 millones de muertes directamente provocadas por la diabetes.

El alto número de personas con este padecimiento ha obligado a la Organización Mundial de la Salud, (OMS) a declararla como un problema de salud pública mundial. Y por ello se ha efectuado un programa para los individuos que padecen esta patología la misma que tiene como misión (3)prevenir esta enfermedad siempre que sea posible y, cuando no lo sea, reducir al mínimo sus complicaciones y mejorar la calidad de vida.

El objetivo general del Programa de Diabetes de la OMS es mejorar la salud estimulando y apoyando la adopción de medidas eficaces de vigilancia, prevención y control de la diabetes y de sus complicaciones, especialmente en los países de ingresos bajos y medios. (3).El Atlas de Diabetes, titulado así, asegura que a pesar de un mejor conocimiento y nuevos avances en el tratamiento de la diabetes tipo 1 y 2, y la prevención de la diabetes tipo 2, cada edición muestra un aumento incesante del número de personas con esta enfermedad. (4)

La diabetes tipo 2 es el tipo de diabetes más común, y ha aumentado junto a los cambios culturales y sociales. En los países de renta alta hasta un $91 \%$ de adultos con la enfermedad tienen diabetes tipo 2. La FID (Federación internacional de Diabetes) estima que 193 millones de personas con diabetes no están diagnosticadas y tienen, por tanto, un mayor riesgo de desarrollar complicaciones. (4)

Por estas razones, las 42 Asambleas Mundial de la Salud del 29 de Mayo de 1989, emitió la Resolución WAH 42.36, en la que se insta a los Estados Miembros a determinar la importancia nacional de la Diabetes, aplicar medidas poblacionales locales para prevenir y controlar la DM insulinoresistente, promover colaboraciones interestatales para el adiestramiento y
Periodo. Enero - Junio 2020

Vol. 2, Nro. 1, Publicado 2020-06-30

educación continuada sobre las acciones cotidianas que conlleva el tratamiento de las personas con Diabetes, así como las acciones de salud pública, estableciendo un enfoque integrado a nivel de la comunidad. (5)

En Cuba, desde 1975 el Instituto Nacional de Endocrinología elaboró un Programa Nacional de Atención Integral al Diabético. Este Programa recibió una mayor prioridad en 1992 cuando se definieron los Objetivos, Prioridades y Directivas para el año 2000 (OPD-2000), estableciendo como meta la disminución de la mortalidad por diabetes en un $15 \%$ en el grupo de población de 15- 64 años y posteriormente se ratificó la decisión de priorizar dicho Programa, aún en las condiciones económicas difíciles del llamado "Período Especial" de la década de los 90, asignado al Instituto Nacional de Endocrinología y la Comisión Nacional de Diabetes la tarea de impulsar, en el contexto del desarrollo del Programa de Enfermedades No Transmisibles el progreso de dicho Programa. (3)

La asociación latinoamericana de diabetes estima que nuestro país tiene un número de casos con individuos que padecen diabetes de 20 a 79 años de edad con un total de 563,840 individuos, con una prevalencia de acuerdo a la OMS de $6.89 \%$, con un número de muertes anuales de 5,492 la misma que demanda gastos anuales por persona de \$335.41, Y los costos por las complicaciones \$22.500. (6) De tal manera que existe un total de casos no diagnosticados de 259,360 y un incremento en el número de casos por año de 19,000. (7) En Ecuador, diabetes están afectando a la población con tasas cada vez más elevadas. Según la encuesta ENSANUT (encuesta nacional de salud y nutrición), la prevalencia de diabetes en la población de 10 a 59 años es de $1.7 \%$. Esa proporción va subiendo a partir de los 30 años de edad, y a los 50, uno de cada diez ecuatorianos ya tiene diabetes. La alimentación no saludable, la inactividad física, el abuso de alcohol y el consumo de cigarrillos, son los cuatro factores de riesgo relacionados directamente con las enfermedades no transmisibles, entre ellas la diabetes. (8)

La misma que demuestra que la prevalencia de la obesidad está aumentando en todos los grupos de edad. 3 de cada 10 niños en edad escolar presenta sobrepeso y obesidad. 1 de cada 4 niños en edad preescolar es pequeño para su edad y el porcentaje del sobrepeso se ha duplicado en las últimas tres décadas. 2 de cada 3 ecuatorianos entre los 19 y 59 años tiene sobrepeso y obesidad, lo que constituye un serio problema de salud pública. (8)

En el año 2008, en Ecuador se registraron más de 3.500 defunciones por diabetes en los hospitales públicos, siendo la principal causa de fallecimientos. La prevalencia se incrementó de 63 a 488 por 100 mil habitantes entre 1999 y 2009, notificándose en 2009 un total de 68.635 casos. La provincia de Santa Elena es la que reporta la prevalencia más alta seguida de Cañar, Manabí y El Oro. (9)

Dentro de la estrategia nacional para las enfermedades crónicas no transmisibles, el Ministerio de salud pública 


\section{HIGIA DE LA SALUD}

(MSP) cuenta con acciones de alta prioridad para garantizar la prevención, diagnóstico oportuno, atención, seguimiento clínico y tratamiento el cual está disponible en todas las unidades de atención, desde la insulina inyectable hasta los medicamentos hipoglucemiantes, cuyos principales usuarios son ciudadanos adultos y adultos mayores. (10)

Según lo que expresan las estrategias del MSP con respecto a los programas de diabetes nos centraremos en realizar el análisis y respectivos para verificar la garantía de estas estrategias, además de comprobar su cumplimiento y la forma en cómo interfiere en sus integrantes. Ya que un programa bien diseñado podría producir importantes reducciones en la mortalidad y comorbilidades asociadas.

Sin embargo, la federación Internacional de Diabetes en su séptima edición expresa que (4) se necesita más educación para mejorar el diagnóstico y el control de todos los tipos de diabetes y para integrar los cambios de estilo de vida que reducirán el aumento de la diabetes tipo 2. Mientras que los programas de educación pueden ayudar a mejorar el control en las personas con diabetes, la educación sanitaria pública es necesaria a nivel poblacional para fomentar cambios en el comportamiento para prevenir la diabetes tipo 2 .

Es importante considerar el componente emocional de los pacientes, debido a que algunos niegan la gravedad de su enfermedad e ignoran la necesidad de auto cuidarse y descuidan el régimen dietético, la medicación, el control de peso y cuidados personales. Otros se vuelven agresivos y pueden exteriorizar su agresividad rechazando el tratamiento o bien la interiorizan deprimiéndose; muchos desarrollan dependencias psicológicas y necesitan que otros cuiden de ellos, sin adoptar un papel activo en su propio cuidado. (11)

El programa nacional de salud para personas con diabetes tiene como finalidad mejorar la calidad de vida de sus pacientes, además de ayudar a sobrellevar la enfermedad y evitar complicaciones la cual comprende, informar mediante educación continua sintomatología, complicaciones, manejo y autocontrol de la patología.

El equipo de salud multidisciplinario principalmente los niveles básicos de atención primaria en salud tienen un rol importante en la prevención de enfermedades tales como la diabetes y de esta manera la enfermería tiene diferentes acciones preventivas y promocionales que dirigen diferentes factores que favorecen el autocuidado y prevención de complicaciones y una de ellas es la educación continua promoviendo un estilo de vida saludable y disminuyendo la morbimortalidad prematura.

Tomando en cuenta estos antecedentes nacen expectativa en el presente trabajo de investigación, ya que a través del conocimiento práctico se puede evidenciar que el paciente diabético a pesar de estar informado acerca de la diabetes autocontrol y autocuidado; sin embargo, no los aplica en su totalidad, debido a ello surgen una serie de complicaciones.
Periodo. Enero - Junio 2020

Vol. 2, Nro. 1, Publicado 2020-06-30

También, esta problemática se ha podido evidenciar durante las prácticas e internado de enfermería en la Atención Primaria de Salud, asistiendo a personas diagnosticadas con diabetes mellitus tipo 2, comprobando que además del inapropiado control y seguimiento de los usuarios diabéticos, también influye la situación económica que no permite la adquisición de los medicamentos para el tratamiento en algunos casos, situación desfavorable para mantener la calidad de vida de los usuarios y la estabilidad familiar, escenario que también incide en la salud emocional.

Sin embargo, el presente trabajo también se justifica por la presencia de pacientes con diagnóstico de diabetes mellitus tipo 2 que acuden al programa de control del centro de salud Chirijos, por lo que es necesario realizar la investigación. Realizando la verificación del impacto de las estrategias establecidas y como están se ponen o no en práctica en sus integrantes diabéticos y si mediante conocimientos palpan o no la realidad. Hay medidas que se llevan a cabo con el equipo multidisciplinario de salud para disminuir la incidencia de sus factores de riesgos y posteriores complicaciones, pero aun así un elevado porcentaje de pacientes hace caso omiso a estas situaciones que a lo largo de su vida afectan y repercuten la calidad de vida.

El adecuado control metabólico en nuestros diabéticos disminuye la incidencia de las complicaciones micro y macrovasculares (estudio UKPDS3), pero el control metabólico obtenido en ellos dista mucho de ser óptimo, pues según diversos estudios suele ser deficiente, aunque variable, y se detecta una infrautilización de medicación para el control de los FRCV (Factores de riesgo relacionados con la calidad de vida). El estudio ELIPSE, realizado en nuestra provincia, concluyó que el grado de control de los FRCV en diabéticos tipo 2 en Atención Primaria (AP) no garantiza una prevención cardiovascular correcta, y aconsejó un uso más intensivo del tratamiento farmacológico. (12)

Los criterios de control metabólico adecuado en pacientes diabéticos según las sociedades científicas como la Asociación Mundial de diabéticos (ADA) son: glucemia basal $<110 \mathrm{mg} / \mathrm{dl}$, glucemia postprandial 130 $180 \mathrm{mg} / \mathrm{dl}$, hemoglobina glicosilada menor de 7\%, presión arterial sistólica/diastólica $<130 /<80$, colesterol total $<185 \mathrm{mg} / \mathrm{dl}$, HDL-colesterol $>40 \mathrm{mg} / \mathrm{dl}$, LDLcolesterol $<100 \mathrm{mg} / \mathrm{dl}$, triglicéridos $<150 \mathrm{mg} / \mathrm{dl}$, no fumar y realizar ejercicio físico de tipo aeróbico al menos 150 minutos/semana (12)

Los datos citados a continuación corresponden a investigaciones realizadas por los autores: Valle Coronado, Vázquez Luis, María López, González Elena, Martín Ruiz, José Miguel, Real Isidoro, Magdalena Sánchez, Ordóñez Carmen y Silveira García en la que se realizó una evaluación en resultados de salud del proceso diabetes tipo 2, la cual tenía como Objetivo analizar la asociación entre el desarrollo del proceso de diabetes tipo 2 y la calidad de vida relacionada con la salud. En la que se utilizó un diseño de estudio descriptivo transversal y se tomó una selección por 


\section{HIGIA DE LA SALUD}

muestreo aleatorio simple entre los pacientes registrados en el proceso DM2 con un total de 143 personas, se recogieron datos por medio de los registros y entrevista personal para el cuestionario de CVRS (calidad de vida relacionada con la salud) SF-36. En conclusión, la CVRS es mala en los diabéticos tipo 2 a pesar de tener buenos indicadores en el proceso por lo cual debería incluirse la CVRS como estándar del proceso DM2. (13) Existe consenso en que el control del paciente diabético requiere usualmente varios fármacos para el control de los FRCV (Factores de riesgo cardiovasculares): antagonistas del sistema renina-angiotensina (SRA-A), antiagregantes en muchos casos y estatinas. También sabemos que el desconocimiento de la propia enfermedad influye desfavorablemente en el cumplimiento terapéutico y éste a su vez en el grado de control. (14)

La presente investigación se pretende realizar en la parroquia Chirijos, perteneciente al Cantón Portoviejo, la misma que según los resultados del último censo poblacional en esta parroquia existen 2.716 habitantes. La mayoría se dedica a la agricultura y a la comercialización de productos agrícolas como la yuca,
Periodo. Enero - Junio 2020

Vol. 2, Nro. 1, Publicado 2020-06-30

café, tagua, caña, naranja, plátano, papaya, mango y también productos maderables como guachapelí, amarillo, moral, algarrobo. El rescate de la cultura Son sitios pertenecientes a esta parroquia El tigre, Roncón, El Limón, Cañales y Jesús María.

Metodología

Para el presente trabajo investigativo se llevó a cabo mediante un estudio de tipo descriptivo, analítico, retrospectivo que consiste en recopilar una gran cantidad de datos de acción rápida mediante la historia clínica de los pacientes que ayuda en la toma de decisiones, y utilizando el análisis y la síntesis de los métodos empíricos y teóricos, para lo que se aplicó una encuesta de conocimientos sobre la enfermedad y conocimiento de autocuidado, también se aplicó el test de findrisk a fin de determinar el resultado de los controles en las personas con diabetes tipo 2 , la muestra de obtuvo mediante criterios de inclusión y exclusión, se tomó como criterio de inclusión a los pacientes que conforman y asisten al programa de diabetes, excluyendo a quienes no asisten a los controles mensuales programados.

\section{Resultados}

\begin{tabular}{|l|c|c|}
\hline Automonitoreo glucemia/glucosuria & Frecuencia & Porcentaje \\
\hline $\mathrm{Si}$ & 2 & 5 \\
\hline $\mathrm{No}$ & 38 & 95 \\
\hline Interpreta automonitoreo glucémico-glucosúrico & 2 & 5 \\
\hline $\mathrm{Si}$ & 38 & 95 \\
\hline $\mathrm{No}$ & & \\
\hline $\mathrm{Monitorea} \mathrm{y} \mathrm{cuida} \mathrm{sus} \mathrm{pies}$ & 31 & 77 \\
\hline $\mathrm{Si}$ & 9 & 23 \\
\hline No & & \\
\hline Previene/trata Hipoglucemia & 10 & 25 \\
\hline $\mathrm{Si}$ & 30 & 75 \\
\hline No & & \\
\hline Es miembro de una asociación de diabetes & 40 & 100 \\
\hline $\mathrm{Si}$ & 0 & 0 \\
\hline No & & \\
\hline ¿Con que frecuencia asiste al club de diabéticos? & 39 & 98 \\
\hline Mensual & 0 & 0 \\
\hline Una Vez Cada 3 Meses & 0 & 0 \\
\hline Seis Veces Al Año & 1 & 3 \\
\hline A Veces & $\mathbf{4 0}$ & $\mathbf{1 0 0}$ \\
\hline
\end{tabular}

Tabla 1. Indicadores de seguimiento y control

Fuente: Encuesta aplicada a personas con diabetes del programa del CS. Chirijos

A continuación, podemos corroborar que los pacientes en un $93 \%$ tienen conocimientos respecto a que si no llevan un manejo adecuado de la glicemia estos valores aumentaran, pero pese a esto se puede evidenciar en sus controles que sus valores son elevados. 
ISSN 2773-7705

HIGÍA DE LA SALUD

Periodo. Enero - Junio 2020

\begin{tabular}{|c|c|c|}
\hline La mantequilla contiene principalmente: & Frecuencia & Porcentaje \\
\hline Proteínas & 0 & 0 \\
\hline Azúcar & 0 & 0 \\
\hline Grasa & 40 & 100 \\
\hline Minerales y Vitaminas & 0 & 0 \\
\hline $\mathrm{No} \mathrm{Se}$ & 0 & 0 \\
\hline \multicolumn{3}{|l|}{ El arroz contiene principalmente: } \\
\hline Proteínas & 0 & 0 \\
\hline Azúcar & 0 & 0 \\
\hline Grasa & 34 & 85 \\
\hline Minerales y Vitaminas & 1 & 3 \\
\hline $\mathrm{NoSe}$ & 5 & 12 \\
\hline \multicolumn{3}{|l|}{$\begin{array}{l}\text { Cuál de los siguientes alimentos puede comer en las cantidades que } \\
\text { usted desee sin peligro de que le aumente su azúcar: }\end{array}$} \\
\hline Manzana & 1 & 3 \\
\hline Pepino & 38 & 94 \\
\hline Carne & 0 & 0 \\
\hline Miel & 1 & 3 \\
\hline No Se & 0 & 0 \\
\hline \multicolumn{3}{|l|}{$\begin{array}{l}\text { Si no le apetece tomar leche para desayunar. ¿Por qué otro alimento lo } \\
\text { puede sustituir? }\end{array}$} \\
\hline Tomando más pan & 0 & 0 \\
\hline Tomando más frutas & 34 & 85 \\
\hline Tomando una porción de queso & 0 & 0 \\
\hline No se & 6 & 15 \\
\hline Total & 40 & 100 \\
\hline
\end{tabular}

Tabla 2. De conocimiento de Dietético y nutricional

Fuente: Encuesta aplicada a personas con diabetes del programa del CS. Chirijos

En cuanto a conocimientos de complicaciones que pueden aparecen en la diabetes el $95 \%$ de los usuarios respondieron que no sabían y el 5\% restante dijo que la presencia de acetona en su orina se encuentra siempre en los diabéticos. Es indicio de su desconocimiento respecto a factor tan importante que puede desencadenar Cetoacidosis diabéticas misma que pone en riesgo la vida del diabético, proceso que ocurre cuando el hígado convierte la grasa en un impulsor llamado cetona que hace que la sangre se vuelva ácida.

En cuanto a los cambios que produce la diabetes en los órganos el $55 \%$ dijo que no produce ningún cambio en los pulmones y el $45 \%$ dijo no sé. Evidentemente un poco menos de la mitad de los miembros de este programa desconoce los cambios y complicaciones que pueden surgir en los órganos por su enfermedad.

\begin{tabular}{|l|l|l|}
\hline & Femenino & Masculino \\
\hline Enero 2018 & $191 \mathrm{mg} / \mathrm{dl}$ & $177 \mathrm{mg} / \mathrm{dl}$ \\
\hline Junio 2018 & $194 \mathrm{mg} / \mathrm{dl}$ & $127 \mathrm{mg} / \mathrm{dl}$ \\
\hline Enero 2019 & $182 \mathrm{mg} / \mathrm{dl}$ & $189 \mathrm{mg} / \mathrm{dl}$ \\
\hline
\end{tabular}

Tabla 3. Media de los niveles de glucosa 2018-2019

Análisis realizado a los pacientes por doce meses

Se aprecia que el programa público no ha logrados cambios importantes al cabo de un año, luego revisar los resultados de control metabólico se aprecia tambien que el género femenino logra menos control que el masculino.

\section{Discusión.}

Según el Modelo de Atención Integral en Salud MAIS (2012) (15)para lograr los objetivos propuesto es preciso instar educación continua por parte del personal de salud mejorando la forma en como trasmiten conocimiento para su mayor interpretación entre los usuarios que conforman el programa respecto a los sucesos más importantes que ellos deben saber en el manejo de su enfermedad y autocuidado. 


\section{HIGIA DE LA SALUD}

Se requiere de manera urgente se hagan adaptaciones en cuanto a la estructura del programa porque se están cumpliendo insuficientemente los objetivos para el cual fue creado.

\section{Conclusiones}

La percepción del impacto de la calidad del programa y control de diabetes mellitus se relacionó con la responsabilidad de salud y control de los miembros del programa. Mediante las fichas clínicas se determinó que el nivel de glicemia no es el apropiado y mucho menos óptimo, no llega a la mitad de los beneficiados que asisten, además existe una ponderación más elevada de riesgo de complicaciones en el sexo femenino, los usuarios tienen un déficit de conocimiento respecto al autocuidado, evolución, control de la enfermedad y sus complicaciones. Además, se precisa prever de Bibliografía
Periodo. Enero - Junio 2020

Vol. 2, Nro. 1, Publicado 2020-06-30

intervenciones de mayor impacto de forma urgente y necesaria para ser más efectivos en el control glicémico. Se demuestra que pese a las diversas actividades y objetivos planteados en el programa de diabetes esta no dan los resultados esperados pese a las diversas actividades que realiza el equipo de salud.

Al conocer el impacto del programa público en el control de diabetes mellitus 2, y resultados es recomendable que se realicen un seguimiento en cada uno de los hogares de los pacientes , trabajar en conjunto con los miembros de sus familias y comunidad aplicando un apropiado plan de acción para diabéticos enfatizando la importancia en el apoyo para el automanejo y control de la glicemia, haciendo mayor énfasis en los adultos de sexo femenino quienes son los que presentan valores considerablemente elevados.

1. Salud OMdl. Informe mundial sobre la diabetes. Mundial. , Salud; 2016.

2. Insulclok. [Online].; 2017 [cited 201901 28. Available from: https://insulclock.com/la-diabetes-en-cifras/.

3. OMS. [Online].; 2018 [cited 201902 10. Available from: https://www.who.int/diabetes/goal/es/.

4. Diabetes FId. Atlas de la Diabetes. Federación Internacional de Diabetes ; 2015. Report No.: 144.

5. Diabetes PNd. Programa Nacional de Diabetes. [Online].; 2016 [cited 201902 10. Available from: http://www.sld.cu/galerias/pdf/sitios/diabetes/programa_nacional_de_diabetes.pdf.

6. El telegrafo. [Online].; 2018 [cited 201901 13. Available from: https://www.eltelegrafo.com.ec/noticias/sociedad/6/programa-atencion-integral-diabeticos.

7. Diabetes ALd. Guías ALAD sobre el Diagnóstico,Control y Tratamiento de la diabetes tipo 2 con medicina basada en evidencia. ALAD. 2013 Nov.

8. ECUADOR O. [Online].; 2018 [cited 201901 28. Available from: https://www.paho.org/ecu/index.php?option=com_content\&view=article\&id=1400:la-diabetes-un-problemaprioritario-de-salud-publica-en-el-ecuador-y-la-region-de-las-americas \&Itemid=360.

9. OPS ECUADOR. [Online].; 2018 [cited 201901 29. Available from: https://www.paho.org/ecu/index.php?option=com_content $\& v i e w=a r t i c l e \& i d=251$ :dia-mundialdiabetes\&Itemid $=360$.

10. MSP. implementa unidades de atención integral para el pie diabético en 10 hospitales.[Online].; 2018 [cited 2019 01 13. Available from: https://www.salud.gob.ec/msp-implementa-unidades-de-atencion-integral-para-el-piediabetico-en-10-hospitales/.

11. Investigacion A. Capacidad de autocuidado en pacientes diabéticos que asisten a consulta externa. Sincelejo, Colombia. Investigacion Cientifica. Pereira: Fundación Universitaria del Área Andina Colombia; 2013. Report No.: 26.

12. Migallón PDS. Control Metabólico en Pacientes Diabéticos Tipo 2: grado de Control y nivel de Conocimientos. Revista Clínica de Medicina de Familia. 2011 Feb; 4(1).

13. Valle Coronado Vázquez LMLGEMR. Evaluación en resultados de salud del proceso diabetes tipo 2. De Investigacion. Huelva Espana:, Unidad de Gestión Clínica de Ayamonte; 2016.

14. Sánchez PD. Control Metabólico en Pacientes Diabéticos Tipo 2: grado de Control y nivel de Conocimientos (Estudio AZUER). Revista Clinica de Medicina Familiar. 2016 Feb; 4(1).

15. Ministerio de Salud Publica. [Online].; 2019 [cited 201901 12. Available from: https://www.salud.gob.ec/ministerio-de-salud-prevencion-y-autocuidado-son-claves-para-controlar-la-diabetes/.

16. INEC. Instituto nacional de estadisticas y censo. [Online].; 2017 [cited 201901 12. Available from: http://www.ecuadorencifras.gob.ec/diabetes-segunda-causa-de-muerte-despues-de-las-enfermedades-isquemicasdel-corazon/.

17. Fundacion Española del Corazon. [Online].; 2018 [cited 201901 13. Available from: https://fundaciondelcorazon.com/prevencion/riesgo-cardiovascular/diabetes.html.

18. ELSEVIER. EDUCACIÓN EN DIABETES. Revista Médica Clínica Las Condes. 2016 Mar; 27(2). 
19. CLINIC M. Foundation for Medical Education and Research. [Online].; 2019 [cited 201901 20. Available from: https://www.mayoclinic.org/es-es/diseases-conditions/type-2-diabetes/symptoms-causes/syc-20351193.

20. Diseases NIoDaDaK. NIH. [Online].; 2019 [cited 201901 20. Available from: https://www.niddk.nih.gov/healthinformation/informacion-de-la-salud/diabetes/informacion-general/sintomas-causas.

21. Candela JM. ¿Cuáles son los factores de riesgo para desarrollar diabetes mellitus tipo 2. ; 2015.

22. Adeslas. [Online].; 2018 [cited 201901 21. Available from: https://www.prevencion.adeslas.es/es/diabetes/masprevencion/Paginas/factores-diabetes.aspx.

23. Coello C. REDACCION MEDICA. [Online].; 2016 [cited 201901 23. Available from: https://www.redaccionmedica.ec/secciones/salud-publica/ecuador-tiene-10-de-prevalencia-de-diabetes-mellitus89013.

24. Bonilla ME. Guía de Práctica Clínica Diabetes Tipo 2. MSP; 2017.

25. Barcelona HSJdD. SJD. [Online].; 2019 [cited 201901 23. Available from: Hospital Sant Joan de Déu Barcelona.

26. Francisco Javier García Soidán FMG. Fisterra.com. [Online].; 2017 [cited 201901 28. Available from: https://www.fisterra.com/diabetes/ficha.asp?acceso=978E90BA78DB55B7D32F42A9A9\&idFicha=93\&token=6 c575650ac6950c84bbf0b5faaa3639f.

27. EE.UU BNdMdl. MedlinePlus. [Online].; 2019 [cited 201901 28. Available from: https://medlineplus.gov/spanish/ency/article/007429.htm.

28. Diagnostics AM. Magazine soluciones para la diabetes. [Online].; 2019 [cited 201901 28. Available from: https://www.solucionesparaladiabetes.com/magazine-diabetes/guia-practica-actividad-fisica-diabetes/.

29. Romero A. Cuidate Plus. [Online].; 2015 [cited 201901 28. Available from: https://cuidateplus.marca.com/enfermedades/digestivas/diabetes/2015/11/12/cuatro-pilares-autocuidadodiabetico-97227.html.

30. OMS. Información sobre el Programa de Diabetes. [Online].; 2019 [cited 201901 28. Available from: https://www.who.int/diabetes/goal/es/.

31. [Online].

32. Alés RMea. Auxiliares de Enfermería del Servicio Navarro de Salud Osasunbidea. Segunda Edición ed. Madrid: MAD, S. L.; 2006.

33. Cardona R. Control metabolico. Madrid: Federacion de diabeticos; 2018.

34. Dionisio Guardiola ACLM. Control Metabólico en Pacientes Diabéticos Tipo 2: grado de Control y nivel de Conocimientos. Clínica de Medicina de familia. 2014 febrero; vol.4 (no.1 ).

35. protocolo. Diabetes Mellitus tipo 2. Protocolo de salud. ; 2017. 